\title{
HEMATOMA EPIDURAL ESPONTÂNEO
}

\author{
RELATO DE DOIS CASOS
}

\begin{abstract}
OSMI HAMAMOTO*, HIROSHI NAKANO*, NILTON E. GUERREIRO*, JOYCE M. A. BIZZACCHI**, VENÂNCIO P. DANTAS FILHO***, EDMUR FRANCO CARELLI*** JOSÉ JORGE FACURE***
\end{abstract}

\begin{abstract}
RESUMO - Os hematomas epidurais espontâneos são raramente descritos na literatura. Estão associados a doenças infecciosas do crânio, distúrbios da coagulação, malformações vasculares da dura-máter e metástases na calota craniana. Os autores relatam dois casos de hematomas epidurais espontâneos de diferentes etiologias e discutem aspectos relacionados à hemostasia.
\end{abstract}

PALAVRAS-CHAVE: hematoma epidural, hematoma espontâneo, hemostasia.

\section{Spontaneous epidural hematoma: report of two cases}

ABSTRACT - Spontaneous epidural hematomas are rarely described in literature. They are associated with infectious diseases of the skull, coagulation disorders, vascular malformations of the dura-mater and metastasis to the skull. The authors report two cases of spontaneous epidural hematoma of different etiologies, and study parameters of hemostasis.

KEY WORDS: epidural hematoma, spontaneous hematoma, hemostasis.

Os hematomas epidurais (HED) são considerados lesões secundárias a traumatismo craniencefálico e sua incidência varia de 0,2 a $30 \%$ de acordo com a literatura ${ }^{1-3}$. Cerca de 83 a $95 \%$ dos casos estão associados a fraturas de crânio $^{2-5}$; porém, quando não há história ou sinais de traumatismo e na ausência de fraturas de crânio, pode-se dizer que o HED ocorreu espontaneamente ${ }^{5}$. Alguns casos de HED espontâneo têm sido relatados, associados a doenças infecciosas (sinusites e otite média aguda) ${ }^{5-11}$, malformações vasculares da dura-máter ${ }^{5,12,13}$, distúrbios da coagulação ${ }^{14,15} \mathrm{e}$ metástases na calota craniana ${ }^{16}$. Neste artigo apresentamos dois casos de HED espontâneo, um deles associado a distúrbios da coagulação e o outro a pansinusite. Aspectos etiológicos dos HED espontâneos são discutidos.

\section{RELATO DOS CASOS}

Caso 1 - LA, 12 anos, sexo feminino, com insuficiência renal crônica devida a infecções urinárias de repetição, por bexiga neurogênica secundária a mielomeningocele corrigida no primeiro dia de vida. Foi submetida a transplante renal, houve moderada perda sanguínea, recebendo cerca de $400 \mathrm{ml}$ de concentrado de hemácias no intra-operatório. Após o transplante foi mantida em UTI; no $3^{\circ}$ dia pós-operatório, sem qualquer história de trauma, tornou-se irritadiça com cefaléia intensa na região parietal direita.

Cerca de 4 horas após o início dos sintomas a paciente encontrava-se na escala de coma de Glasgow com

*Disciplina de Neurocirurgia, Faculdade de Medicina de Marília (FAMEMA); **Departamento de Hematologia, Hospital de Clínicas - Faculdade de Ciências Médicas, Universidade Estadual de Campinas (FCM/ UNICAMP); ***Departamento de Neurocirurgia, Hospital de Clínicas, FCM/UNICAMP. Aceite: 7-março-1998.

Dr. Osmi Hamamoto - Rua 21 de Abril 263 - 17515-220 Marília SP - Brasil. 
7 pontos, hemiparesia esquerda e anisocoria, com midríase à direita. Seus exames mostravam - hemoglobina (Hb) 6,6 g/dl; hematócrito $(\mathrm{Ht}) 20,1 \%$; plaquetas $=154.000 / \mathrm{mm}^{3}$; tempo de protrombina (TP) $20 \mathrm{seg}$ (normal 13 seg.); atividade de protrombina (AP) 49\%; tempo de trombloplastina parcial ativado (TTPA) 41 seg. (normal 46,3 seg.); tempo de trombina (TT) 10 seg. (normal 9,5 seg.); RX de crânio: ausência de fraturas; Tomografia
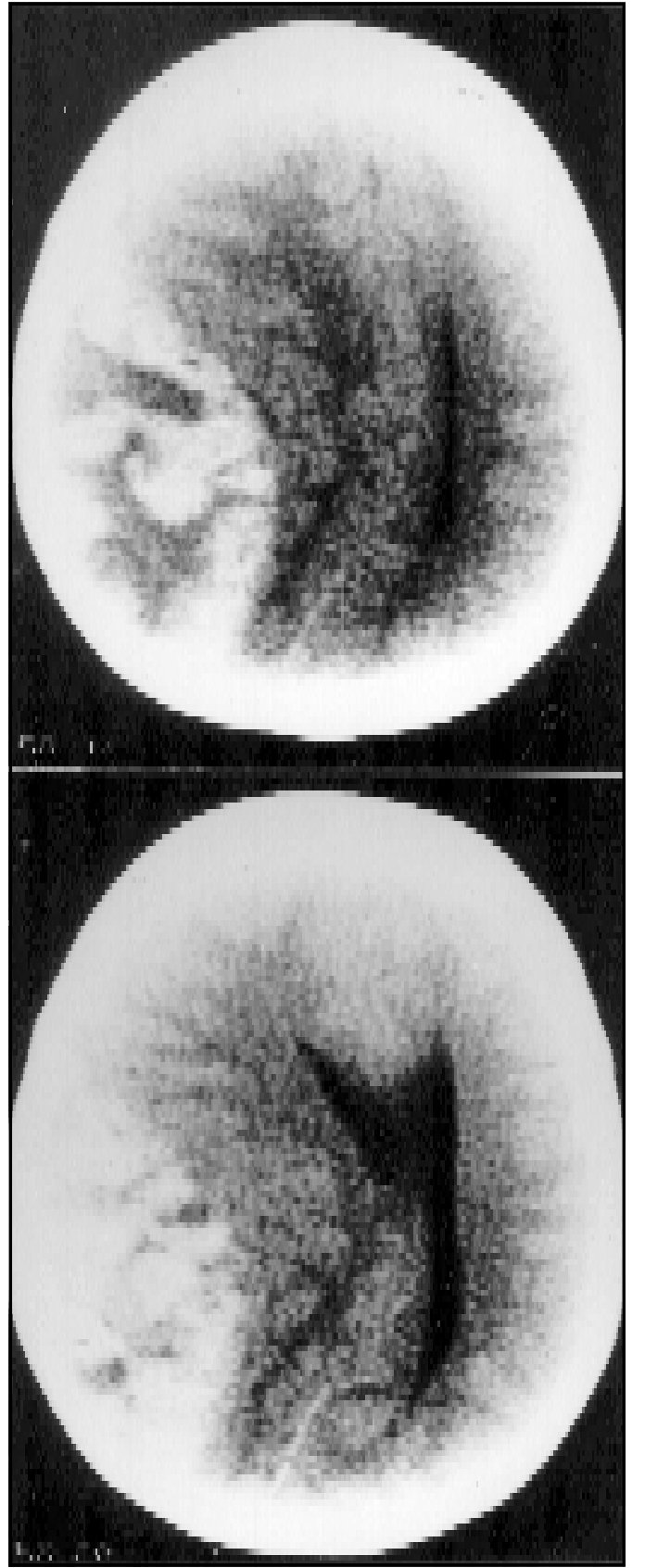

Fig 1. CT de crânio sem contraste mostrando hematoma epidural parieto-occipital à direita. computadorizada de crânio: coleção extra-axial, biconvexa, com áreas hipo e hiperdensas na região parieto-occipital direita, compatível com hematoma epidural agudo (Fig 1).

A paciente foi submetida a cirurgia de emergência, sendo realizada craniotomia parietooccipital direita com drenagem do HED. Durante a cirurgia nenhuma evidência de trauma foi vista no couro cabeludo ou na calota craniana e, após a drenagem, nenhuma malformação vascular foi encontrada na dura-máter. No leito do hematoma não identificamos qualquer grande vaso sanguíneo ao qual pudéssemos atribuir a origem do sangramento; havia apenas sangramento difuso. Recebeu no intra-operatório $400 \mathrm{ml}$ de concentrado de hemácias.

Após a cirurgia mantivemos a paciente sob sedação e ventilação mecânica; no $4^{\circ}$ dia pós operatório realizamos CT de controle que mostrou melhora acentuada do padrão tomográfico e pequena coleção laminar residual. Evoluiu de maneira satisfatória e recebeu alta da UTI no $7^{\circ}$ dia após a drenagem do hematoma, consciente com pupilas isocóricas e fotorreagentes e com leve hemiparesia esquerda. No intuito de esclarecermos a etiologia do sangramento, no $2^{\circ}$ dia após a drenagem do hematoma, realizamos testes laboratoriais para avaliar os sistemas de coagulação e fibrinólise: plaquetas $155.000 / \mathrm{mm}^{3}$; TP 14,2 seg. (normal 13,7 seg.); AP 84\%; TTPA 41 seg. (normal 46,3 Seg.); TT 16 seg. (normal 14,3 seg.); fator V $155 \%$ (normal $122 \% \pm 32$ ); fibrinogênio (FIBR) $381 \mathrm{mg} / \mathrm{dl}$ (Normal 190 a $450 \mathrm{mg} / \mathrm{dl}$ ); plasminogênio (PLM) 3,20 U/ml (normal 2,49 a 3,57 U/ml); produtos de degradação do fibrinogênio e fibrina (PDF ) 10-40 $\mu \mathrm{g} /$ $\mathrm{ml}$ (normal na $1^{\text {a }}$ diluição $<10 \mu / \mathrm{ml}$; ; tempo de lise da euglobulina (TLE) $335 \mathrm{~min}$ (normal 286 $\pm 74 \mathrm{~min}$ ); área de lise em placa de fibrina (ALPF) $64 \mathrm{~mm}^{2}$ (normal $79 \pm 21 \mathrm{~mm}^{2}$ ); alfa-2 macroglobulina (A2MG) 100\% (normal 88\% \pm 22); pré-albumina $86,9 \%$ (normal $108 \% \pm 18$ ); pré-calicreína plasmática $0,411 \mathrm{U} / \mathrm{ml}$ (normal $0,530 \pm 0,150 \mathrm{U} / \mathrm{ml})$

No seguimento de 18 meses após a cirurgia a paciente apresentava-se sem sequelas neurológicas, porém faleceu em consequência de complicações da insuficiência renal.

Caso 2 - CRPS, 15 anos, sexo masculino, com história de quadro gripal há 1 semana; há 5 dias iniciou febre e cefaléia leve. Há 3 dias da 
sua internação começou apresentar obstrução nasal, rinorréia amarelada com estrias de sangue e cefaléia intensa, pulsátil, hemicraniana esquerda. Não havia qualquer história de traumatismo craniano e nem relatos de distúrbios da coagulação.

O paciente apresentava-se consciente, sonolento, orientado no tempo e espaço, na escala de coma de Glasgow com 14 pontos, sem déficit motor e com pupilas isocóricas e fotorreagentes. À fundoscopia havia papiledema bilateral. A rinoscopia mostrou tecido necrótico com exposição da cartilagem, na porção posterior de ambas fossas nasais, mais centralizado no septo nasal posterior, e ausência de neoplasias. Seus exames préoperatórios mostraram - Hb 12,7 g/dl; Ht 39,7\%; Leucócitos 13.800/mm³; PL 218.000/mm³; TP 17,9 seg. (normal $15,2 \mathrm{seg}$ ); TTPA 26,9 seg. (normal 26,6 seg.); TT 12,5 seg. (Normal $10 \mathrm{seg}$.); RNI 1,22; FIBR $261 \mathrm{mg} \%$ (normal 190-450 mg\%); fator V 100\% (normal 70-160\%). RX de crânio: não mostrava sinais de fratura. RX de seios da face: pansinusite. CT de crânio: coleção hiperdensa, extra-axial, biconvexa na região frontal esquerda, com desvio da linha média para a direita, compatível com hematoma epidural.

Foi submetido a craniotomia frontal esquerda e drenado o HED. Durante a cirurgia nenhuma evidência de trauma foi encontrada no couro cabeludo e na calota craniana. O HED estava firmemente aderido à duramáter e em algumas áreas de sua superfície havia tecido fibrinoso amarelado. Após a retirada do hematoma não observamos comunicação do espaço epidural com os seios paranasais e nem malformações vasculares durais, porém a dura-máter sangrava difusamente. $\mathrm{O}$ hematoma foi enviado para exame anatomopatológico.

Após a cirurgia o paciente evoluiu de maneira satisfatória e teve alta hospitalar, depois de ser tratada a sinusite aguda, sem qualquer sequela neurológica. No seguimento de 4 meses após a cirurgia encontrava-se sem queixas e de volta às suas atividades. $\mathrm{O}$ exame anatomopatológico do material revelou tratar-se de hematoma epidural em organização, incipiente, associado a material fibrino-purulento. Com o propósito de esclarecer a etiologia do sangramento, no $30^{\circ}$ dia pós-operatório foram dosados os seguintes fatores: fator VIII 156\% (normal $60-150 \%$ ); fator IX 56\% (normal 50-150\%); fator X 56\% (normal 70-120\%); fator XI 151\% (normal 60-140\%); fator XII $151 \%$ (normal $=60-140 \%$ ).

\section{DISCUSSÃO}

A maioria dos autores acredita que para um HED ocorrer, há necessidade de haver lesão vascular e descolamento da dura-máter da superfície interna do $\operatorname{osso}^{2,3,17}$, condições estas encontradas no traumatismo craniencefálico.

A presença de fratura de crânio nos HED é quase que obrigatória, principalmente quando se trata de adolescentes, sua incidência variando de 83 a $95 \%{ }^{2-5}$. Quando frente a um HED sem história de traumatismo craniencefálico e ausência de fratura de crânio, podemos inferir que se trata de hematoma epidural espontâneo 5 . Este tipo de hematoma associa-se a quatro categorias etiológicas: infecções pericranianas (sinusites, otites), malformações vasculares durais, metástases na calota craniana e distúrbios da coagulação.

Em relação às infecções pericranianas, os casos apresentados na literatura estão associados a sinusite frontal ${ }^{7,8,11}$ ou otite média ${ }^{5,6,9,10}$. Nesta categoria dois mecanismos são propostos para explicar a ocorrência do HED. No primeiro, o processo infeccioso se estenderia para a superfície externa da dura-máter causando enfraquecimento da parede dos vasos epidurais (vasculite), levando a ruptura deles e acúmulo de sangue. Este mecanismo é justificado por evidências radiológicas, operatórias e histológicas, encontrando-se contiguidade do processo infeccioso às estruturas ósseas (osteíte focal) ${ }^{5-7}$. No segundo mecanismo, um progressivo descolamento da dura-máter da superfície óssea interna, em consequência ao acúmulo excessivo de exsudato ou ar da área infectada, levaria à lesão vascular, proporcionando o desenvolvimento do $\mathrm{HED}^{9}$. No Caso 2, embora não encontrássemos contiguidade entre os seios paranasais e o espaço epidural, o exame anatomopatológico deixou claro que houve extensão da infecção para a superfície externa da dura-máter e talvez tenha sido através do osso etmóide que ela tenha se propagado.

As malformações vasculares da dura-máter são lesões raras e a ocorrência de HED secundário a elas é pouco frequente, pois raramente apresentam sangramento e, quando este ocorre, é para os espaços subdural e subaracnóide ${ }^{5,13}$. Gallagher e Browder ${ }^{18}$, relataram um caso de malformação arteriovenosa dural que cursou com HED. 
Em relação às metástases na calota craniana, Nakagawa e col. ${ }^{16}$ descreveram o caso de um paciente de 52 anos que desenvolveu HED occipital supra e infratentorial consequente ao sangramento de metástase na calota craniana de carcinoma hepatocelular; porém, esta ocorrência é rara.

Os distúrbios da coagulação congênitos ou adquiridos ${ }^{14}$ constituem, junto com as infecções pericranianas, as duas grandes categorias de patologias às quais os HED espontâneos costumam se associar. Kuwayama e col. ${ }^{15}$ relataram o caso de uma paciente que desenvolveu HED espontâneo bilateral e relacionaram a etiologia do sangramento a hipofibrinogenemia adquirida. Em sua revisão da literatura citam oito casos de HED associados a hemofilia e um caso associado a deficiência de vitamina $\mathrm{K}$.

A literatura nos mostra que, durante grandes cirurgias abdominais, cardíacas, ginecológicas, urológicas e de tumores cerebrais, há ativação da coagulação e consequente ativação secundária do sistema fibrinolítico ${ }^{19-24}$. Isto pode ser evidenciado no Caso 1 , pelo encontro de moderado aumento nos produtos de degradação do fibrinogênio e fibrina (PDF). A avaliação de alguns parâmetros da coagulação em ambos os casos não mostrou alterações importantes que justificassem o desenvolvimento dos hematomas, pois os exames não revelaram deficiência de fatores da coagulação e nem estado de hiperfibrinólise, embora esta última situação possa ser mais uma causa para o desenvolvimento de hematomas epidurais espontâneos.

\section{REFERÊNCIAS}

1. Bullock R, Teasdale G. Surgical management of traumatic intracranial hematomas. Vinken PJ, Bruyn GW, Klawans HL (eds). Handbook of clinical neurology, Vol 57 (head injury). New York: Elsevier, 1990:249-298.

2. Cooper PR. Post-traumatic intracranial mass lesion. In. Cooper PR (ed). Head injury. New York: William \& Wilkins 1987:238-284.

3. Jamieson KG, Yelland JDN. Extradural hematoma: report of 167 cases. J Neurosurg 1968;29:13-23.

4. Chan K, Mann KS, Yue CP, et al. The significance of skull fracture in acute intracranial hematomas in adolescentes: a prospective study. J Neurosurg 1990;72:189-194.

5. Sanchis JF, Orosco M, Cabanes J. Spontaneous extradural hematomas. J Neurol Neurosurg Psychiatry 1975;38:577-580.

6. Clein LJ. Extradural hematoma associated with middle-ear infection. Can Med Assoc J 1970;102:1183-1184.

7. Kelly DL Jr, Smith JM. Epidural hematoma secondary to frontal sinusitis: case report. J Neurosurg 1968;29:67-69.

8. Marks SM, Shaw MDM. Spontaneous intracranial extradural hematoma: case report. J Neurosurg 1982;57:708-709.

9. Novaes V, Gorbitz C. Extradural hematoma complicating middle-ear infection: report of a case. J Neurosurg 1965;23:352-353.

10. Punt J. Chronic extradural hematoma presenting 33 years after penetrating cranial trauma: case report. J Neurosurg 1978;49:103-106.

11. Rajput AJ, Rozdilsky B. Extradural hematoma following frontal sinusitis: report of a case and review of the literature. Arch Otolaryngol 1971;94:83-86.

12. Hasegawa H, Bitoh S, Fujiwara M, et al. Spontaneous intracranial extradural hematoma. Neurol Med Chir (Tokyo) 1983;23:968-971.

13. Urbanibia JF, Silvela J, Soto M. Occipital dural arteriovenous malformations. Neuroradiology 1974;7:57-64.

14. Grabel JC, Sacher M, Rothman AS. Bilateral extradural hematomas in a trombocytopenic infant receiving anticoagulants: case report. Neurosurgery 1989;25:828-830.

15. Kuwayama N, Takahashi S, Sonobe M et al. Spontaneous bilateral extradural hematomas: case report. J Neurosurg 1985;62:139-141.

16. Nakagawa Y, Yoshino E, Suzuki K, et al. Spontaneous epidural hematoma from a hepatocelular carcinoma metastasis to the skull: case report. Neurol Med Chir (Tokyo) 1992;32:300-302.

17. Ford LE, McLaurin RL. Mechanism of extradural hematomas. J Neurosurg 1963;20:760-769.

18. Gallagher JP, Browder EJ. Extradural hematoma: experience with 167 patients. J Neurosurg 1963;29:1-12.

19. Bennett B, Ogston D. Fibrinolytic bleeding syndromes. In Ratnoff OD, Forbes CD (eds). Disordes of hemostasis. Philadelphia: WB Saunders, 1991:327-351.

20. Sawaya R, Ramo OJ, Glass-Greenwalt P, et al. Plasma fibrinolytic profile in patients with brain tumors. Thromb Haemostas 1991;65:15-19.

21. Teufelsbauer H, Proidl S, Havel M et al. Early activation of hemostasis during cardiopulmonary bypass: evidence for thrombin mediated hyperfibrinolysis. Thromb Haemostas 1992;68:250-252.

22. Ygge J. Changes in blood coagulation and fibrinolysis during the postoperative period. Am J Surg 1970;119:225-232.

23. Aranda A, Paramo JA, Rocha E. Fibrinolytic activity in plasma after gynecological and urological surgery. Hemostasis 1988;18:129-134.

24. Facure JJ, Bizzachi JAM, Carelli EF et al. Estudo da fibrinólise em meningiomas. Arq Bras Neurocirurg 1992;11:182-190. 